Иванов С. Н., Сарилов М. Ю., Просолович А. А., Решетник У. Е.

РАЗРАБОТКА И МОДЕЛИРОВАНИЕ АВТОМАТИЧЕСКОГО ПРИВОДА ЗАПОРНОЙ АРМАТУРЫ

ЛОКАЛИЗАЦИИ ЦЕНТРОБЕЖНОГО НАСОСА

Иванов С. Н., Сарилов М. Ю., Просолович А. А., Решетник У. Е. S. N. Ivanov, M. Y. Sarilov, A. A. Prosolovich, U. E. Reshetnik

РАЗРАБОТКА И МОДЕЛИРОВАНИЕ АВТОМАТИЧЕСКОГО ПРИВОДА ЗАПОРНОЙ АРМАТУРЫ ЛОКАЛИЗАЦИИ ЦЕНТРОБЕЖНОГО НАСОСА

\title{
DESIGN AND MODELING OF THE AUTOMATIC DRIVE OF THE LOCK VALVE LOCALIZATION OF A CENTRIFUGAL PUMP
}

Иванов Сергей Николаевич - доктор технических наук, доцент, профессор кафедры электромеханики Комсомольского-на-Амуре государственного университета (Россия, Комсомольск-на-Амуре); 681013, Хабаровский край, г. Комсомольск-на-Амуре, пр. Ленина, 27. E-mail: Ivanov.sn@email.knastu.ru.

Mr. Sergey N. Ivanov - Dr. habil. in, professor, Electrical Engineering Department, Komsomolsk-on-Amur State University (Russia, Komsomolsk-on-Amur); 681013, Khabarovsk territory, Komsomolsk-on-Amur, 27 Lenin str. E-mail: Ivanov.sn@email.knastu.ru.

Сарилов Михаил Юрьеевич - доктор технических наук, профессор кафедры машин и аппаратов химических производств Комсомольского-на-Амуре государственного университета (Россия, Комсомольск-наАмуре); 681013, Хабаровский край, г. Комсомольск-на-Амуре, пр. Ленина, 27. E-mail: sarilov@knastu.ru.

Mr. Mikhail Y. Sarilov - Dr. habil. in Engineering, Head of Machinery and Equipment of Chemical Industry Department, Komsomolsk-on-Amur State University (Russia, Komsomolsk-on-Amur); 681013, Khabarovsk territory, Komsomolsk-on-Amur, 27 Lenin str. E-mail: sarilov@knastu.ru.

Просолович Алексей Александрович - кандидат технических наук, доцент кафедры систем автоматизированного проектирования Комсомольского-на-Амуре государственного университета (Россия, Комсомольск-на-Амуре); 681013, Хабаровский край, г. Комсомольск-на-Амуре, пр. Ленина, 27; тел.: + 7 (914) 185-93-59. E-mail: office@knastu.ru.

Mr. Alexey A. Prosolovich - PhD in Engineering, Associate Professor, Computer-aided design systems Department, Komsomolsk-on-Amur State University (Russia, Komsomolsk-on-Amur); 681013, Khabarovsk territory, Komsomolsk-on-Amur, 27 Lenin str.; tel.: + 7 (914) 185-93-59. E-mail: office@knastu.ru.

Решетник Ульяна Евгеньевна - магистр кафедры машин и аппаратов химических производств Комсомольского-на-Амуре государственного университета (Россия, Комсомольск-на-Амуре); 681013, Хабаровский край, г. Комсомольск-на-Амуре, пр. Ленина, 27; тел.: + 7 (914) 185-65-41. E-mail: Reshetnik @)gmail.com.

Ms. Ulyana E. Reshetnik - master' s degree student, Machinery and Equipment of Chemical Industry Department, Komsomolsk-on-Amur State University (Russia, Komsomolsk-on-Amur); 681013, Khabarovsk territory, Komsomolsk-on-Amur, 27 Lenin str.; tel.: + 7 (914) 185-65-41. E-mail: Reshetnik @gmail.com.

Аннотация. В статье рассматриваются вопросы разработки и моделирования автоматического привода запорной арматуры локализации центробежного насоса, используемого в технологическом процессе транспортирования углеводородных носителей. Приведены основные достоинства приводов на базе электромеханических преобразователей переменного тока. Показан алгоритм моделирования с использованием программного комплекса T-Flex. Рассмотрена физическая реализация преобразователя и приведены рекомендации по его проектированию. Обоснованы критерии работоспособности применительно к объекту исследования. Показано, что синтез привода и его системы управления может быть проведен на основе известных в машиностроении и теории управления подходов. Повышение эффективности и надёжности привода обеспечивается интеграцией исполнительного устройства непосредственно в приводной механизм. Приведены результаты моделирования и проектирования базовых элементов привода, адаптирована машиностроительная методика оценки надёжности исполнительного элемента привода, предназначенного для использования в качестве автоматизированного механизма возвратно-поступательного перемещения оборудования транспортного назначения.

Summary. The article deals with the design and modeling of the automatic drive of the lock valve localization of a centrifugal pump used in the technological process of transportation of hydrocarbon carriers. The main advantages of the drives on the basis of electromechanical converters of alternating current are provided. The algo- 
rithm of modeling using t-Flex software complex is shown. The physical implementation of the Converter is considered and recommendations for its design are given. The criteria of efficiency in relation to the object of study are grounded. It is shown that the synthesis of the drive and its control system can be carried out on the basis of approaches well-known in machine-building and control theory. Efficiency and reliability improvement of the drive is provided by the actuator integration directly into the water mechanism. The results of modeling and design of the basic elements of the drive are presented, the engineering technique of assessing the reliability of the executive element of the drive intended for use as an automated mechanism of reciprocating movement of transport equipment is adapted.

Ключевые слова: проектирование, моделирование, центробежный насос, работоспособность, автоматический привод.

Key words: design, simulation, centrifugal pump, operability, automatic drive.

\section{УДК 621.3 .019}

\section{Введение}

Транспортирование вязких жидкостей традиционно осуществляется по трубопроводным магистралям с помощью насосных установок центробежного типа. Теоретически поток перекачиваемой жидкости характеризуется объёмным расходом и средней по сечению трубы скоростью. Расход является одной из основных характеристик потоков жидкости и определяется количеством жидкости, которое перемещается через поперечное сечение трубопровода в единицу времени. При движении реальных жидкостей часть механической энергии движения необратимо превращается в тепловую. Эта часть энергии в традиционных устройствах называется потерями энергии, обусловленными вязкостью жидкости. Часть мощности, затрачиваемая насосом на транспортирование вязкой жидкости, компенсирует потери энергии, связанные с потерей давления и потерей напора. При этом гидравлические потери давления (напора) делят на потери давления на трение при движении жидкости, равномерно распределённой по всей длине трубы, и местные потери, сосредоточенные на сравнительно коротких участках трубопроводов, обусловленных местными изменениями формы канала. Типичными примерами местных сопротивлений являются участки резкого расширения (диффузоры) и сужения (конфузоры) трубопровода, места слияния и разделения потоков, трубопроводная аппаратура (вентили, клапаны, задвижки, дроссели и т. п.). Характерной особенностью движения жидкости через местные сопротивления является образование вихрей в потоке, что вызывает значительные потери энергии (давления, напора).

Анализ состава энергетического оборудования, обеспечивающего технологический процесс транспортирования нефтепродуктов, показывает, что одним из самых энергоёмких видов оборудования являются насосные агрегаты, состоящие из центробежного насоса и электродвигателя, причём единичная мощность применяемых для привода насосов электродвигателей находится в диапазоне 5,5...1250 кВт и соответственно определяет мощность и стоимость всего агрегата. Практика эксплуатации насосных установок показывает, что по ряду причин (высокое использование, неравномерный график нагрузки, разброс параметров транспортируемой жидкости и т. д.) это оборудование характеризуется повышенным индексом технического риска.

В этой связи задача обеспечения экономичной и надёжной транспортировки углеводородных носителей актуальна не только при разработке новых удалённых мест добычи, но и при модернизации существующих магистралей. Одним из направлений повышения эксплуатационных характеристик таких объектов является создание и разработка новых эффективных устройств, обеспечивающих автономность их технического сопровождения.

В качестве таких устройств, позволяющих автоматизировать процессы вывода насосного оборудования из технологического цикла для ремонта, профилактики или замены, можно рассматривать высоконадёжные интеллектуальные привода́ на основе асинхронных двигателей с короткозамкнутым ротором. Это связано с такими возможностями привода, как

- получение тяговых усилий, ограниченных только прочностью запорной арматуры; 
Иванов С. Н., Сарилов М. Ю., Просолович А. А., Решетник У. Е.

РАЗРАБОТКА И МОДЕЛИРОВАНИЕ АВТОМАТИЧЕСКОГО ПРИВОДА ЗАПОРНОЙ АРМАТУРЫ

ЛОКАЛИЗАЦИИ ЦЕНТРОБЕЖНОГО НАСОСА

- уникальные регулировочные характеристики (возможность обеспечения и точного поддержания линейной скорости запорного элемента в диапазоне $0,001 \ldots 10 \mathrm{M} / \mathrm{c}$ );

- повышение коэффициента полезного действия и эффективности оборудования в целом при эксплуатации и техническом обслуживании;

- принципиальное улучшение массогабаритных и стоимостных показателей;

- возможность дистанционного автоматического управления для труднодоступного и удалённого оборудования.

Основой разработки являются результаты реализации опытного образца в качестве привода автоматизированной системы комплексной безопасности [1]. Полученные при этом практические результаты подтвердили целесообразность и перспективность использования разработки в качестве силового привода с гарантированными показателями безотказности и работоспособности.

Важно отметить, что устройство может адаптироваться под экстремальные внешние воздействия в зависимости от условий эксплуатации. В разработке реализован принцип электромагнитного преобразования электрической энергии в управляемое линейное перемещение исполнительного механизма. Теоретически устройство может быть использовано в качестве элементов гидросооружений, на транспортных объектах, в строительном и технологическом оборудовании и т. п.

\section{Описание научно-практической проблемы}

Проектирование и моделирование автоматического привода (АП) при недетерминированном графике нагрузки представляет сложную научную задачу, требующую одновременного учёта динамики системных электромагнитных, механических, гидравлических и тепловых процессов. Для определения диапазона выполнимости АП и исключения энергетической избыточности целесообразно использование современных методов планирования эксперимента и проектирования с привлечением интегрированных CAD- и CAE-систем, являющихся универсальным средством вариативного проектирования и многофакторной оптимизации сложных физических процессов [2 - 4].

Использование САПР предполагает разработку геометрии и создание сборочной модели АП. Это требует обоснования компоновки, выбора расчётных моделей для вычислительного эксперимента САЕ-методами. На начальном этапе проектирования при большом числе или отсутствии адекватной информации о влиянии отдельных параметров целесообразно использование методов планирования эксперимента. Нахождение субоптимального варианта требует исследования различных вариантов автоматического привода и, соответственно, обеспечения возможности варьируемой геометрической модели.

\section{Моделирование привода}

Общая структурная схема автоматического привода рассмотрена в работе [1]. Формально разработка параметрической модели вращающейся части привода необязательна, так как она стандартизована, унифицирована и соответствует габариту базового асинхронного двигателя. По справочным данным [5], находятся размерные соотношения базовых элементов длина $l$ привода и диаметр $d$, которые определяют область проектирования. Базовой деталью привода является тело вращения «Корпус». Геометрическая 3D-модель «Корпус» выполнена с использованием CAПР T-Flex CAD («Топ-Системы», г. Москва).

Объект компьютерного проектирования - параметрическая сборочная 3D-модель привода. В базовой детали «Корпус» позиционируется сборочная единица «Тяга», которая изображена на рис. $1, a$.

Для оценки расположения поверхностей и формы элементов АП, проверки конструкционной совместимости, определения эргономических свойств методом послойного синтеза на 3D-принтере Dimension 3D Printers from Stratasys из пластика изготовлена модель базовых элементов АП, приведённая на рис. 1,6 .

Физическая реализация АП показана на рис. 2 . 


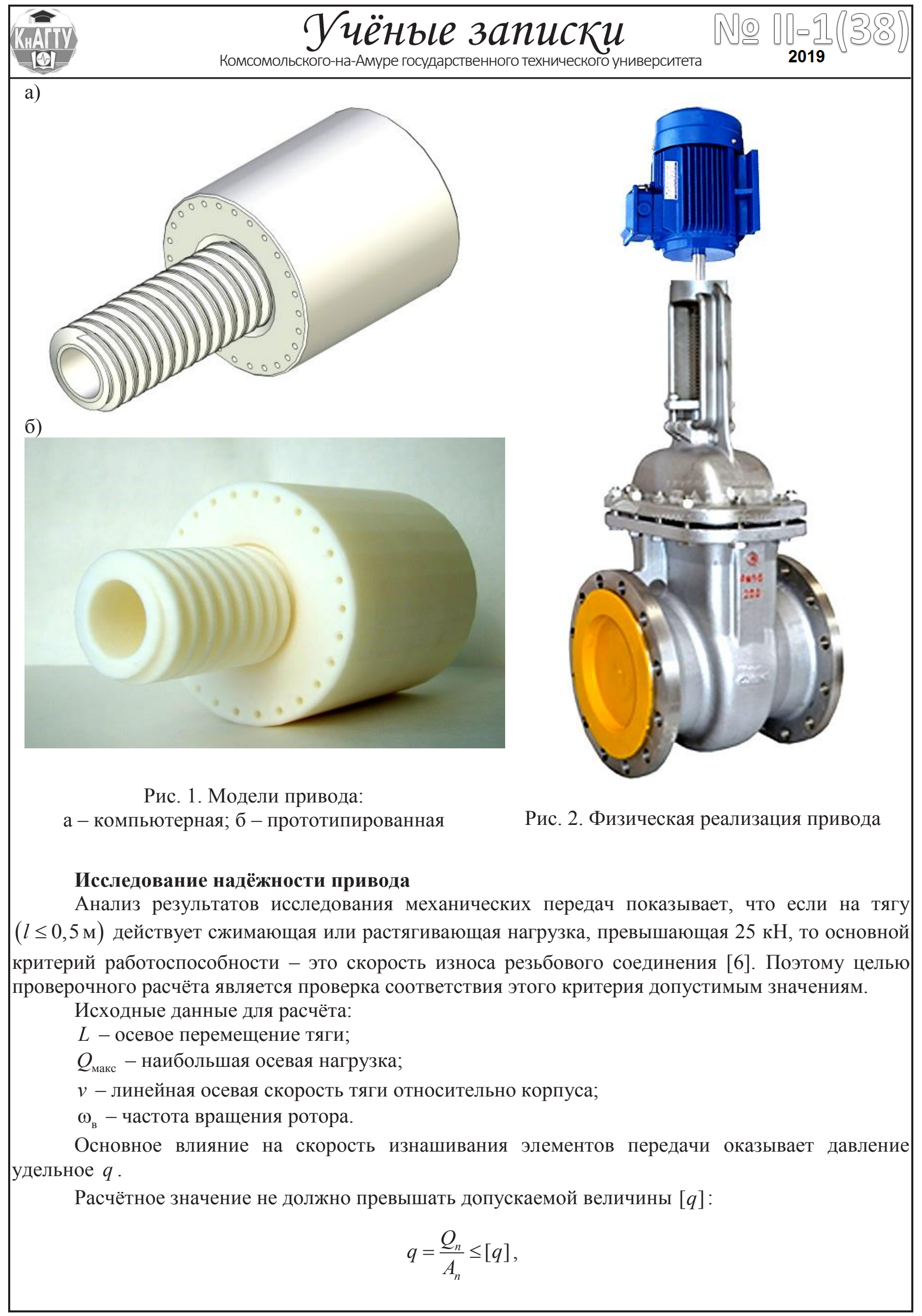


Иванов С. Н., Сарилов М. Ю., Просолович А. А., Решетник У. Е.

РАЗРАБОТКА И МОДЕЛИРОВАНИЕ АВТОМАТИЧЕСКОГО ПРИВОДА ЗАПОРНОЙ АРМАТУРЫ

ЛОКАЛИЗАЦИИ ЦЕНТРОБЕЖНОГО НАСОСА

где $Q_{n}$ - нормальное изгибное усилие; $A_{n}$ - теоретическая площадь сопряжения взаимно перемещающихся элементов.

Давление равномерно распределённой по виткам нагрузки составит:

$$
\frac{Q_{n}}{A_{n}}=\frac{Q}{z \cdot A},
$$

где $Q$ - осевая сила тяги, $\mathrm{H} ; A$ - проекция площади рабочей поверхности витка на плоскость, перпендикулярную оси тяги; $z$ - рабочее число витков.

Условием износостойкости является выражение

$$
q=\frac{Q}{z \cdot \pi \cdot d_{2} \cdot H_{1}} \leq[q]
$$

где $d_{2}$ - средний диаметр резьбы; $H_{1}$ - рабочая высота профиля; $[q]$ - нормативное давление, МПа.

Это выражение содержит три проектных параметра и имеет несколько решений. При приближённых расчётах принимают:

- относительную глубину $\psi_{h}=H_{1} / p$ (трапецеидальные резьбы $\psi_{h}=0,5$, упорные $\left.\psi_{h}=0,75\right) ; \psi_{H}$ предварительно принимается в пределах $1,2 \ldots 2,5$; большее значение принимается для силового привода;

- приведённую высоту ротора $\psi_{h}=H_{p} / d_{2}, H_{\mathrm{P}}$ - высота ротора.

Полная высота резьбовой части ротора $H_{\mathrm{P}}=z \cdot P$, где $P$ - шаг резьбы. Средний диаметр резьбы $d_{2} \geq \sqrt{\frac{Q}{\pi \cdot \psi_{h} \cdot \psi_{H} \cdot[q]}}$.

Последующий расчёт включает выбор материалов резьбовой части ротора и тяги; нахождение нормированного давления $[q]$ и допускаемых напряжений; относительной глубины резьбы $\psi_{h}$, относительной высоты ротора $\psi_{\mathrm{H}}$; среднего диаметра резьбы.

При сжимающей тягу осевой силе $Q$ проверяется продольная устойчивость на изгиб. Гибкость тяги рассчитывается по выражению $\lambda=\frac{4 \cdot \mu \cdot L}{d_{3}}$, где $\mu-$ коэффициент, учитывающий способ закрепления опор (шарнирные опоры $\mu=1$ ).

Критическое напряжение гибкости $\lambda_{1}$ соответствует потери устойчивости и равно пределу пропорциональности.

По формуле Эйлера расчёт на устойчивость ведётся для критической силы, если $\lambda>\lambda_{1}$ :

$$
Q_{\text {кр }}=\frac{\pi^{2} \cdot E \cdot I}{(\mu \cdot L)^{2}},
$$

где $E$ - модуль упругости материала тяги; $I=\pi \cdot d_{3}^{4} / 64-$ момент инерции в поперечном направлении сопряжения.

Расчёт на устойчивость ведётся для критической силы при $\lambda<\lambda_{1}$ ведётся по формуле

$$
Q_{\mathrm{kp}}=\frac{\pi \cdot d_{3}^{4}}{4} \cdot(a-b \cdot \lambda),
$$




\section{Учёные записки \\ Комсомольского-на-Амуре государственного технического университета

где $a$ и $b$ - экспериментальные коэффициенты. Требуемый коэффициент запаса устойчивости $s_{y}$ должен быть не ниже детерминированного $\left[s_{y}\right]$ :

$$
s_{y}=\frac{Q_{\mathrm{kp}}}{Q} \geq\left[s_{y}\right] .
$$

Рекомендуемое значение $\left[s_{y}\right]=3 \div 4$.

При заданных частоте вращения $\left(\omega_{\text {в }}\right)$ и осевой скорости тяги $(v)$ число заходов резьбы рассчитывается по формуле $n=\frac{2 \cdot \pi \cdot v}{\omega \cdot P}$.

Для расчёта угла подъёма резьбы используется выражение $\psi=\operatorname{arctg} \frac{P_{h}}{\pi \cdot d^{2}}$,где величина хода $P_{h}$ определяется произведением числа заходов на шаг.

С учётом угла профиля приведённый к расчётным условиям коэффициент трения в резьбе определяется по формуле $f^{\prime}=\frac{f}{\cos \alpha / 2^{\prime}}$, где $f$ - коэффициент трения материалов; $\alpha$ - угол профиля.

Приведённый к расчётным условиям угол трения $\rho^{\prime}=\operatorname{arctg} f^{\prime}$.

Момент трения $M_{p}$ обусловлен действующей осевой силой и зависит от конструктивного исполнения опоры.

Если наружный и внутренний диаметры $D_{0}$ и $d_{0}$, для кольцевой опоры момент трения составит $M_{\text {оп }}=\frac{1}{3} \cdot Q \cdot f \cdot \frac{D_{0}^{3}-d_{0}^{3}}{D_{0}^{2}-d_{0}^{2}}$, где $f-$ коэффициент трения на поверхности.

На кручение устойчивость тяги проверяется при одновременном действии напряжений растяжения и сжатия исходя их значения $\sigma_{\ni}=\sqrt{\left(\frac{4 \cdot Q}{\pi \cdot d_{3}^{2}}\right)^{2}+3 \cdot\left(\frac{M}{0,2 \cdot d_{3}^{3}}\right)^{2}} \leq[\sigma]_{\text {p(ж) }}$, где $M-$ тангенциальный крутящий момент; $[\sigma]_{\mathrm{p}(ж)}-$ нормируемое при растяжении (сжатии) напряжение.

Условие отсутствия среза резьбы проверяется по выражению $\tau_{\text {ср }}=\frac{Q}{\pi \cdot d \cdot z \cdot b} \leq[\tau]_{\text {ср }}$, где $b$ для упорной резьбы $0,736 P$; для трапецеидальной $0,634 P$; $[\tau]_{\mathrm{cp}}-$ допускаемое напряжение.

Допускаемые для материала напряжения резьбовой части ротора назначаются по машиностроительным рекомендациям, приведённым в работе [7].

В качестве дополнительного критерия работоспособности устойчивость при продольном изгибе может быть принята, если приведённая длина тяги выше 0,7 м или сжимающие осевые усилия не превышают $15 \div 20$ кН.

\section{Модель объекта управления}

Процесс автоматической локализации насосного агрегата из технологического цикла комплекса для транспортировки обеспечивает система управления с использованием интеллектуальных подходов, реализация которой наиболее целесообразна в виде базы знаний $\{u, x\}$, обеспечивающей возможность учёта управляющих параметров и внешних случайных возмущений.

Анализ используемых подходов показывает, что для АП необходим учёт трёх управляемых параметров (тяговое усилие, линейная скорость перемещения тяги, давление транспортируемого носителя), приводящий к построению базы знаний в виде системы уравнений:

$$
\left\{\begin{array}{l}
x_{1}, x_{2}, x_{3}=f\left(u_{1}\right) \\
x_{1}, x_{2}, x_{3}=f\left(u_{2}\right)
\end{array}\right.
$$


Иванов С. Н., Сарилов М. Ю., Просолович А. А., Решетник У. Е.

РАЗРАБОТКА И МОДЕЛИРОВАНИЕ АВТОМАТИЧЕСКОГО ПРИВОДА ЗАПОРНОЙ АРМАТУРЫ

ЛОКАЛИЗАЦИИ ЦЕНТРОБЕЖНОГО НАСОСА

где $x_{1}, x_{2} x_{3}$ - выходные параметры; $u_{1}, u_{2}$ - управляющие параметры.

Если требования к системе управления относительно невысоки, то количество перекрещивающихся связей между выходными параметрами может быть уменьшено заменой одного из них через остальные параметры. В общем случае система уравнений (1) приводится к виду

$$
\left\{\begin{array}{l}
x_{1}\left(x_{2}, x_{3}\right), x_{2}\left(x_{1}, x_{3}\right), x_{3}\left(x_{1}, x_{2}\right)=f\left(u_{1}\right) \\
x_{1}\left(x_{2}, x_{3}\right), x_{2}\left(x_{1}, x_{3}\right), x_{3}\left(x_{1}, x_{2}\right)=f\left(u_{2}\right)
\end{array}\right.
$$

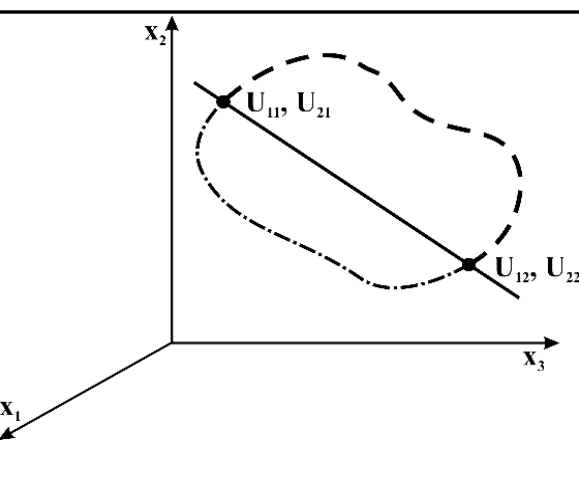

Рис. 3. К определению траектории управления

На рис. 3 условно показаны возможные траектории управления, определяемые входными параметрами $u_{11}, u_{21}$ и $u_{12}, u_{22}$, обеспечивающие требуемые выходные параметры $x_{1}, x_{2}, x_{3}$ (тяговые усилия и линейные скорости, являющиеся выходными параметрами АП).

\section{Заключение}

Результаты разработки и моделирования автоматического привода, предназначенного для запорной арматуры локализации центробежного насоса, полученные с использованием компьютерных 3D-технологий, показывают целесообразность его применения в качестве перспективного энергетического оборудования.

Приведённая методика расчёта критериев работоспособности позволяет производить оценку основных показателей надёжности и долговечности привода. Полученные результаты являются основой для создания различных видов транспортного и промышленного силового оборудования, использующего прецизионные механизмы линейного перемещения и позиционирования.

\section{ЛИТЕРАТУРА}

1. Пат. 2651531 C1 Российская Федерация, МПК ${ }^{7}$ B61L 29/04. Автоматизированная пропускная система / С. Н. Иванов, Ю. С. Иванов, С. Г. Баена, О. С. Амосов; заявитель и патентообладатель Комсомольский-наАмуре гос. техн. ун-т. - № 2017113804; заявл. 20.04.2017; опубл. 19.04.2018. Бюл. № 11. - 2 с.

2. Большаков, В. П. 3D-моделирование в AutoCAD, KOMI1AC-3D, SolidWorks, Inventor, T-Flex: учебный курс / В. П. Большаков, А. Л. Бочков, А. А. Сергеев. - СПб.: Питер, 2011. - 336 с.

3. Алямовский, А. А. SolidWorks Simulation. Как решать практические задачи / А. А. Алямовский. - СПб.: БХВ-Петербург, 2012. - 448 с.

4. SolidWorks 2007/2008. Компьютерное моделирование в инженерной практике / А. А. Алямовский, А. А Собачкин, Е. В. Одинцов [и др.]. - СПб.: БХВ-Петербург, 2008. - 1040 с.

5. Копыловов, И. П. Справочник по электрическим машинам / И. П. Копыловов, Б. К. Клокова. - М.: Энергоатомиздат, 1988. - Т. 1-2.

6. Иванов, М. Н. Детали машин / М. Н. Иванов, В. А. Финогенов. - М.: Высш. шк, 2008. - 408 с.

7. Анурьев, В. И. Справочник конструктора-машиностроителя / В. И. Анурьев. - М.: Машиностроение, 2001. - T. 1-3. 